\title{
Perineural and Vascular Invasion in an Endocrine Mucin-Producing Sweat Gland Carcinoma of the Ear with Associated Mucinous Carcinoma
}

\author{
Neera R. Nathan ${ }^{a} \quad$ Kevin S. Emerick ${ }^{b} \quad$ Mai P. Hoang ${ }^{c} \quad$ Gideon P. Smith $^{a}$ \\ Molly Yancovitz ${ }^{\mathrm{a}, \mathrm{d}}$ \\ a Department of Dermatology, Massachusetts General Hospital, Boston, MA, USA; \\ ${ }^{b}$ Division of Head and Neck Oncology, Department of Otolaryngology, Massachusetts \\ Eye and Ear Infirmary, Boston, MA, USA; ' Department of Pathology, Massachusetts General \\ Hospital, Boston, MA, USA; ${ }^{d}$ Mohs and Dermatologic Surgery Division, Department of \\ Otolaryngology, Massachusetts Eye and Ear Infirmary, Boston, MA, USA
}

\section{Keywords}

Endocrine mucin-producing sweat gland carcinoma - Perineural invasion - Mohs surgery ·

Excision · Radiation

\section{Abstract}

Endocrine mucin-producing sweat gland carcinoma (EMPSGC) is a low-grade, indolent tumor found almost exclusively on the eyelids that may histologically mimic metastatic breast carcinoma. To our knowledge, we present the first case of EMPSGC located on the external ear, and the first case with histologic evidence of vascular and perineural invasion. Due to the aggressive potential of this lesion, wide local excision and adjuvant radiation therapy were performed to help reduce the risk of recurrence.

\section{Case Report}

A 74-year-old woman with no prior history of cutaneous or internal malignancy presented to our dermatology department for a 2-week history of a growing lesion on her right tragus with associated numbness and tingling. Clinical exam revealed a $7-\mathrm{mm}$ firm, pink to violaceous papule (Fig. 1). Shave biopsy showed nests of keratinocytes lacking peripheral palisading, suggestive of the surface of an adnexal neoplasm. The patient was referred for excision to better characterize the lesion. On surgical evaluation, the lesion was tender and felt fixed to the underlying tragus. It measured $2 \mathrm{~cm}$ at the greatest palpable dimension without associated lymphadenopathy. 
Fig. 1. A 7-mm firm, violaceous papule on the right tragus is shown.

Nathan et al.: Perineural and Vascular Invasion in an Endocrine Mucin-Producing Sweat Gland Carcinoma of the Ear with Associated Mucinous Carcinoma

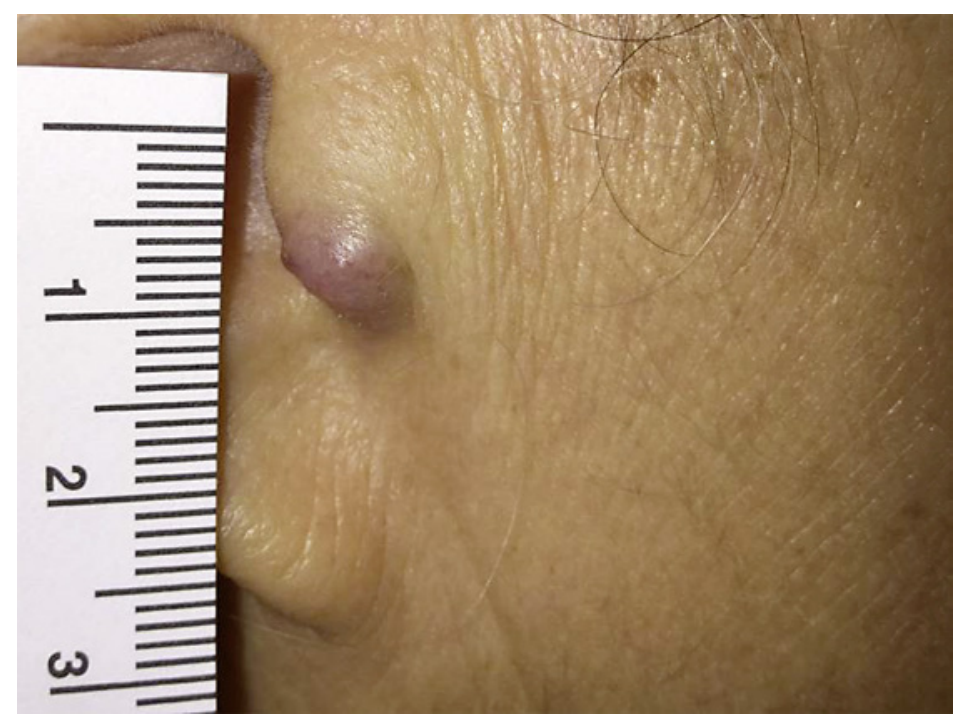

Fig. 2. An infiltrative carcinoma with cribriform architecture and associated mucinous component is noted $(100 \times)$. Vascular invasion is noted in the upper right-hand corner (arrow, 400x) and shown at higher magnification in the inset.

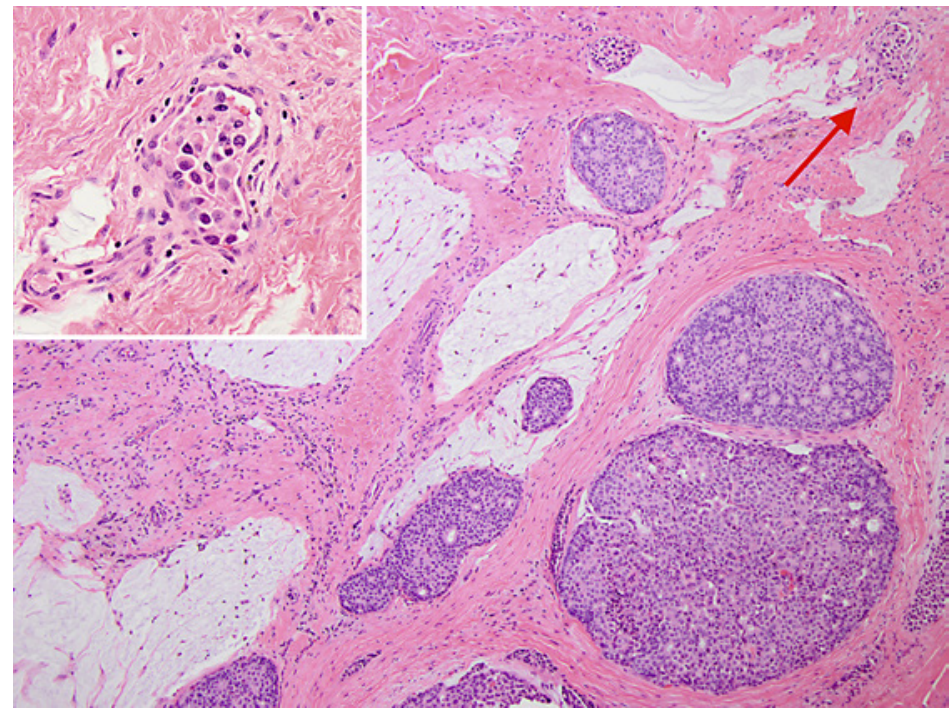

\section{Diagnosis and Clinical Course}

Pathology from incisional biopsy was consistent with an endocrine mucin-producing sweat gland carcinoma (EMPSGC), present at the deep margin. Of note, the patient had no history of breast cancer, and a normal mammogram the month before. Computed tomography of the neck did not show involvement of deeper structures or lymphadenopathy. She underwent excision with 1-cm margins as well as resection of the tragal cartilage. Pathology showed an infiltrative carcinoma with extensive vascular invasion, perineural invasion (PNI), and a focal associated mucinous carcinoma, with infiltrating carcinoma present at the deep margin (Fig. 2, Fig. 3). The tumor was immunoreactive for keratin 7, chromogranin, synaptophysin, androgen receptor, and estrogen receptor - an immunoprofile supportive of EMPSGC. She underwent re-excision of the right preauricular skin and additional medial tragal soft tissue and cartilage which showed residual carcinoma present adjacent to cartilage, all margins were negative for malignancy. Based on the indistinct margins of the tumor, deep invasion to cartilage, and perineural spread, a multidisciplinary team recommended adjuvant 
Fig. 3. Perineural invasion is seen in the lower right-hand corner $(400 \times)$.

Nathan et al.: Perineural and Vascular Invasion in an Endocrine Mucin-Producing Sweat Gland Carcinoma of the Ear with Associated Mucinous Carcinoma

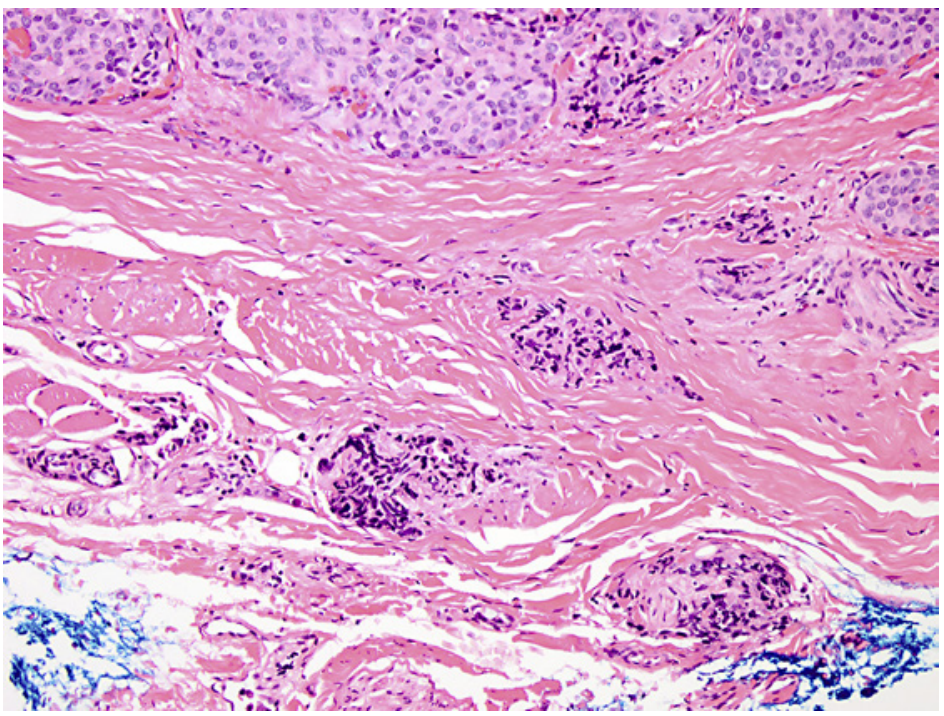

radiation. The patient received a total of 60 Gy of radiation in 30 fractions to the surgical site, ipsilateral parotid gland and neck (level $2 / 3$ lymph nodes). She is being followed clinically every 3 months, with repeat imaging every 6 months. She has had no evidence of recurrence in an 18-month follow-up period since completing her radiation treatments.

\section{Discussion}

EMPSGC is a rare, slow-growing tumor that generally presents as a solitary, asymptomatic nodule on the eyelid of elderly individuals $[1,2]$. Local recurrence may occur following surgical excision [3]; however, there have been no reported cases of lymph node involvement or distant metastases.

EMPSGC is a primary skin tumor characterized by well-defined nodules of uniform polygonal epithelial cells with bland nuclei [3]. Several studies have demonstrated that EMPSGC may be a precursor lesion to invasive mucinous carcinoma in the skin given synchronous presentations [1]. As this tumor is morphologically similar to endocrine ductal carcinoma in situ of the breast, metastatic breast cancer should be considered in the differential diagnosis $[1,3]$.

Prior reports of EMPSGC with or without invasive mucinous carcinoma describe an indolent tumor that lacks vascular invasion or PNI. PNI and lymphovascular invasion have been documented in other sweat gland tumors, which can behave aggressively, and be more likely to recur or metastasize [4, 5]. Treatment for EMPSGC typically consists of tumor extirpation via wide local excision or Mohs surgery [1,2]. Due to high-risk histologic features and deep extension of this preauricular tumor, the patient was treated with wide local excision followed by adjuvant radiation to reduce the risk of recurrence and treat the at-risk regional lymph nodes. While this treatment has not been reported in EMPSGC, surgery followed by adjuvant radiation in adnexal tumors of the head and neck with high-risk features has shown promise to improve locoregional control [5]. 


\section{Statement of Ethics}

This study is a case report and does not include identifiable photographs or any patient identifiers. The manuscript was prepared in compliance with all ethical and confidentiality guidelines and principles.

\section{Disclosure Statement}

The authors have no relevant conflicts of interest to declare.

\section{Funding Sources}

The authors did not receive any funding for this study.

\section{Author Contributions}

Neera R. Nathan made substantial contributions to the acquisition, analysis, and interpretation of data for the work, drafted the work, approved the final version, and agrees to be accountable for all aspects of the work in ensuring that questions related to the accuracy or integrity of any part of the work are appropriately investigated and resolved. Mai P. Hoang made substantial contributions to the analysis and interpretation of data for the work, revised the work for important intellectual content, approved the final version, and agrees to be accountable for all aspects of the work in ensuring that questions related to the accuracy or integrity of any part of the work are appropriately investigated and resolved. Kevin S. Emerick, Gideon P. Smith, and Molly Yancovitz made substantial contributions to the acquisition, analysis, and interpretation of data for the work, revised the work for important intellectual content, approved the final version, and agrees to be accountable for all aspects of the work in ensuring that questions related to the accuracy or integrity of any part of the work are appropriately investigated and resolved.

\section{References}

1 Zembowicz A, Garcia CF, Tannous ZS, Mihm MC, Koerner F, Pilch BZ. Endocrine mucin-producing sweat gland carcinoma: twelve new cases suggest that it is a precursor of some invasive mucinous carcinomas. Am J Surg Pathol. 2005 Oct;29(10):1330-9.

2 Scott BL, Anyanwu CO, Vandergriff T, Nijhawan RI. Endocrine Mucin-Producing Sweat Gland Carcinoma Treated With Mohs Micrographic Surgery. Dermatol Surg. 2017 Dec;43(12):1498-500.

3 Flieder A, Koerner FC, Pilch BZ, Maluf HM. Endocrine mucin-producing sweat gland carcinoma: a cutaneous neoplasm analogous to solid papillary carcinoma of breast. Am J Surg Pathol. 1997 Dec;21(12):1501-6.

4 Cardoso JC, Calonje E. Malignant sweat gland tumours: an update. Histopathology. 2015 Nov;67(5):589-606.

5 Wang LS, Handorf EA, Wu H, Liu JC, Perlis CS, Galloway TJ. Surgery and Adjuvant Radiation for High-risk Skin Adnexal Carcinoma of the Head and Neck. Am J Clin Oncol. 2017 Aug;40(4):429-32. 